\title{
Doppler optical coherence tomography for energy seal evaluation and comparison to visual evaluation
}

\author{
Andrew J. Marques \\ Robnier Reyes \\ Christopher R. Pasarikovski \\ Chaoliang Chen \\ Joel Ramjist \\ Xijia $\mathrm{Gu}$ \\ Victor Yang
}




\title{
Doppler optical coherence tomography for energy seal evaluation and comparison to visual evaluation
}

\author{
Andrew J. Marques, ${ }^{\text {a }}$ Robnier Reyes, ${ }^{\text {a }}$ Christopher R. Pasarikovski, ${ }^{\text {b }}$ \\ Chaoliang Chen, ${ }^{\mathrm{a}}$ Joel Ramjist, ${ }^{\mathrm{a}}$ Xijia Gu, ${ }^{\mathrm{c}}$ and Victor Yang, ${ }^{\mathrm{a}, \mathrm{d}, \mathrm{e},}$ \\ ${ }^{a}$ Ryerson University, Bioengineering and Biophotonics Laboratory, Department of Electrical, \\ Computer, and Biomedical Engineering, Toronto, Ontario, Canada \\ ${ }^{b}$ University of Toronto, Division of Neurosurgery, Department of Surgery, \\ Toronto, Ontario, Canada \\ ${ }^{\mathrm{c}}$ Ryerson University, Department of Electrical, Computer, and Biomedical Engineering, \\ Toronto, Ontario, Canada \\ ${ }^{\mathrm{d} S u n n y b r o o k}$ Health and Sciences Center, Division of Neurosurgery, Toronto, Ontario, Canada \\ ${ }^{\mathrm{e}}$ University of Toronto, Division of Neurosurgery, Faculty of Medicine, Toronto Ontario, Canada
}

\begin{abstract}
Laser energy sealing systems have attracted much attention over the past decade given the general shift in surgical paradigm toward less invasive surgical approaches. Given this, it is paramount to have an objective method with which the quality of energy seals can be evaluated. Current methodologies used for this purpose can be problematic in the evaluation of small vessel seals. A methodology employing Doppler optical coherence tomography (DOCT) for the evaluation of energy seals is introduced. Avian chorioallantoic membrane vessels were subjected to thulium laser irradiation and were then scanned via OCT. Outcomes were classified based on several markers, predominantly the presence or absence of flow postirradiation. Vessel diameter and general morphology were also taken into consideration. Vessels were classified into four groups: seal (29\%), rupture (30\%), partial seal (19\%), and unaffected $(22 \%)$. All vessels were also evaluated visually by a trained neurovascular surgeon, and these visually classified outcomes were compared with DOCT evaluated outcomes. It was found that whether the vessel was considered sealed or not sealed was dependent on the evaluation method ( $p=0.01)$ where visual classification resulted in $18 \%$ more seals than DOCT classification. Further, the specificity of visual classification was found to be strongly dependent on the number of partial seals $(p<0.0001)$. DOCT has shown to be an indispensable method for the evaluation of energy seals not only solely due to its high velocity resolution but also due to valuable microscopic morphological insight regarding the biological mechanisms responsible for energy sealing. () The Authors. Published by SPIE under a Creative Commons Attribution 4.0 Unported License. Distribution or reproduction of this work in whole or in part requires full attribution of the original publication, including its DOI. [DOI: 10.1117/1.JBO.25.3.035003]
\end{abstract}

Keywords: energy sealing; optical coherence tomography; hemostasis; thulium.

Paper 190335R received Sep. 23, 2019; accepted for publication Feb. 20, 2020; published online Mar. 9, 2020; corrected Apr. 9, 2020.

\section{Introduction}

Laser energy sealing is the process by which a laser is used to occlude a blood vessel. It has numerous applications across multiple surgical sectors including tumor devascularization, ${ }^{1,2}$ the treatment of various endolaryngeal maladies, ${ }^{3-5}$ and the treatment of arteriovenous malformations. ${ }^{6,7}$ In all cases, the intent is to mitigate the flow of blood to a body of tissue with minimal collateral damage or simply to achieve hemostasis by ceasing the extravasation of blood. Laser-based systems are an attractive option for the energy sealing of vessels as they offer several advantages over conventional instruments. These include the potential to induce hemostasis in a contactless fashion and the ability to achieve selective tissue interaction with wavelength selection. ${ }^{8}$ In the last decade or so, these systems have attracted more attention,

*Address all correspondence to Victor Yang, E-mail: yangv@ryerson.ca 
given the general shift in surgical paradigm toward the use of less invasive surgical instruments and the general ease with which laser light can be conducted through such instruments. Given this, it is paramount to have an objective method with which vessel seals can be evaluated so that the hemostatic capabilities of laser-based systems can be tested. Current methodologies used for this purpose include burst pressure analysis (BPA), ${ }^{9-11}$ microscopy, ${ }^{12}$ and visual examination. ${ }^{13-16}$ While BPA yields valuable insight as to the strength of seals, it cannot be performed in situ without invasive measures. Microscopic analysis allows for detailed views of the irradiation site and is effective in observing blood flow even in small arteries. However, it relies on an unimpeded view of the vessel, which at times is not granted, given the development of coagulum, and further does not yield depth-resolved information. It is crucial to evaluate the entirety of a vessel cross-section for flow in order to ensure that a vessel has been completely sealed, thus depthresolved information is needed. Visual examination is a rapid method and is ideal in noncritical scenarios where complete cessation of blood flow is not necessary. Despite this, it is highly subjective, making it a poor choice for scientific processes and impractical for vessels below $1 \mathrm{~mm}$ in diameter due to limitations of the human eye.

In this paper, a new modality for the evaluation of laser energy seals is introduced: Doppler optical coherence tomography (DOCT). DOCT imaging features a high sensitivity to flow, typically capable of detecting flows as low as $130 \mu \mathrm{m} / \mathrm{s}$ in tissue. ${ }^{17}$ Clinical applications of DOCT include vasoactive drug screening, hemodynamic monitoring following pharmacological and photodynamic therapy, and mapping cortical hemodynamics. ${ }^{18}$ In a study comparing Doppler ultrasound to CT angiography, the gold standard in vascular flow detection, it was found that Doppler ultrasound correctly detected 50/58 carotid occlusions. ${ }^{19}$ Of the eight false positives (no flow detection when in fact there was flow), seven were cases of near complete occlusion, thus extremely low flow rates, and one was a result of an anatomical anomaly where a sharp 90 deg bend of the vessel occurred in the imaging field resulting in failed Doppler signal acquisition. DOCT has two orders greater velocity resolution than conventional Doppler ultrasound systems ${ }^{17}$ and thus, it is likely to succeed in detecting flows present in near totally occluded vessels. As such, it is reasonable to conclude that DOCT will have a higher sensitivity (i.e., in detecting complete seals) than Doppler ultrasound bringing it closer to the gold standard of CT angiography. The primary limitation of DOCT is its shallow penetration depth, which limits the depth at which velocity information can be resolved to a maximum of $3 \mathrm{~mm}$. This means that DOCT, for the purposes of energy seal evaluation, is only viable for small vessels with diameters below $3 \mathrm{~mm}$. Further, DOCT can only target superficial vessels, vessels that have been surgically exposed, or vessels that have been brought within close proximity of the instrument in laparoscopic/catheter-based cases. Despite this, DOCT offers an objective method for evaluating the quality of energy seals and given its high velocity resolution is capable of detecting minute flows that are otherwise invisible to the eye. Although DOCT does not generate information relating to the strength of energy seals, as with BPA, its potential for rapid in situ assessment of energy seals in small vessels makes it an attractive method for the evaluation of laser sealing systems.

The purpose of this study is to develop a methodology for the evaluation of energy seals induced in small vessels using DOCT. The intent is to use this methodology to evaluate the hemostatic potential of laser-based systems in an objective manner. To the author's knowledge, there have been no studies that employ DOCT for this purpose. In the literature, several studies exist where a laser's ability to induce complete seals in vessels is evaluated visually. Therefore, given the nature of this study, it is valuable to compare visual evaluation with DOCT evaluation such that the accuracy of visual methods in detecting blood flow can be assessed.

\section{System Description}

A thulium fiber laser with 1942-nm output was used in this experiment to seal vessels. It has been reported previously. ${ }^{20}$ The thulium fiber laser is compact, high-powered, tuneable, and lases at a strongly hydrolytic wavelength and thus is especially well suited to medical applications. As can be seen in Fig. 1, imaging was accomplished using a spectral-domain optical coherence tomography (OCT) system with a supercontinuum source (SuperK Extreme from NKT Photonics) centered at $1310 \mathrm{~nm}$. In medical systems, it is important to minimize cost, thus, although a swept 


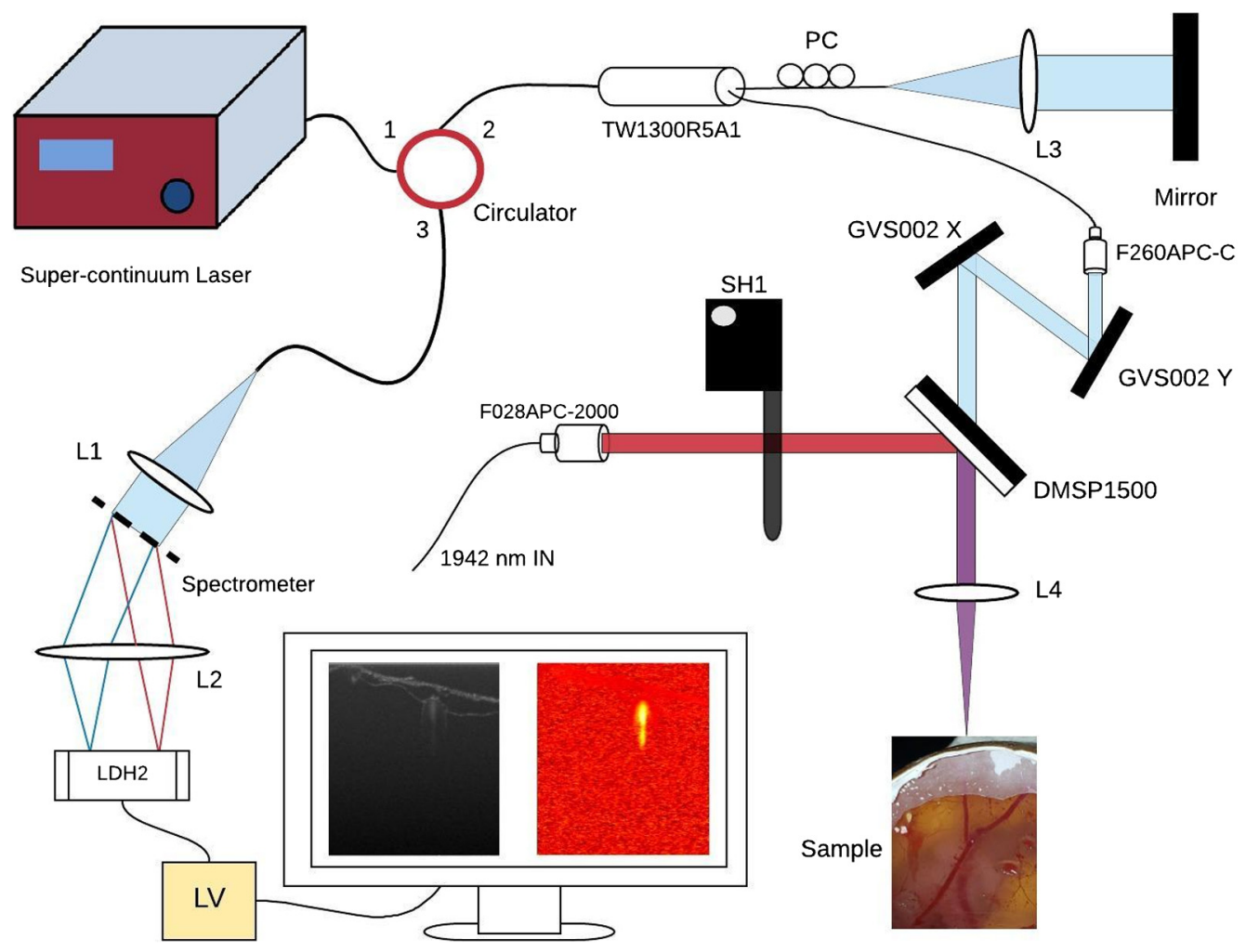

Fig. 1 Optical system diagram. L1-L4, achromatic lenses. A polarization controller is situated in the reference arm of the interferometer (PC). LV, LabVIEW processing block.

source system may have offered better performance, ${ }^{21}$ a spectrometer-based system was employed instead. The fiber-based Michelson interferometer consists of a THORLABS CIR1310-50-APC circulator and a THORLABS TW1300R5A1 beam-splitter to maintain the wide bandwidth of the supercontinuum source. It also includes a three-paddle polarization controller to facilitate polarization matching. At the sample arm, the beam is collimated using the THORLABS F260APC-C collimation package before passing through the GVS002 galvo system for sweeping. The beam was swept in one-dimension over a distance (at the sample) of $6.2 \mathrm{~mm}$. Power balance between the reference and sample arms was carefully done during the calibration phase. Dispersion compensation between the arms was done numerically in software. The interference signal was acquired using a custom spectrometer from P\&P Optica, which has a grating frequency of 892 lines $/ \mathrm{mm}$, a spectral resolution of $0.365 \mathrm{~nm}$, and a wavelength range of 1190 to $1370 \mathrm{~nm}$, achieving an axial resolution of $\sim 6 \mu \mathrm{m}$ in tissue. This in comparison with the resolution of high-frequency ultrasound, which is typically between 50 and $100 \mu \mathrm{m}{ }^{22}$ The camera of the spectrometer (Goodrich LDH2) contains an array of 1024 sensors being sampled at $10,813 \mathrm{~Hz}$ (A-line rate). The sampled signal was processed using a LabVIEW VI through the steps of resampling, FFT, dispersion compensation, and log compression for display. A shortpass dichroic mirror (THORLABS DMSP1500) was used to couple the 1942- and 1300-nm beams. The mirror was fixed at exactly $45 \mathrm{deg}$ using the THORLABS C3P60R. After passing through the mirror, the coupled beam was focused using a $\mathrm{CaF}_{2}$ lens $(f=100 \mathrm{~mm})$. Using a beam profiler, the resulting spot size at focus of the OCT beam was found to be $\sim 70 \mu \mathrm{m}$. At 6.2-mm lateral beam sweep and 1024-A-lines per sweep, this ensured sufficient overlap for the acquisition of Doppler signals. Profilers in the $2-\mu \mathrm{m}$ regime are extremely costly thus spot size of the 1942-nm beam was approximated theoretically using

$$
2 w_{0}=\frac{4 \lambda f}{\pi d},
$$

where $2 w_{0}$ is the diameter of the spot at the sample, $\lambda$ is the wavelength, $f$ is the focal length, and $d$ is the diameter of the spot at the focal lens. ${ }^{23}$ The minimum spot size at the sample was 
approximated to $206 \mu \mathrm{m}$ where $d=1.2 \mathrm{~mm}$. In this work, the effects of spot size on the seal outcome were not considered. The spot size was varied such that a variety of laser irradiation outcomes could be observed. For this reason, a theoretical value for the spot size was sufficient. The velocity resolution of the DOCT system was found to be $112 \mu \mathrm{m} / \mathrm{s}$. For comparison, the minimum detectable blood flow velocity of a 6-MHz Doppler ultrasound system is typically $6.4 \mathrm{~mm} / \mathrm{s} ;{ }^{24}$ however, velocity resolutions of $\sim 100 \mu \mathrm{m} / \mathrm{s}$ have been reported in higher frequency systems. ${ }^{25}$ The velocity resolution of the DOCT system used here was calculated using the minimum detectable phase shift corresponding to an SNR of 25 in the following equation:

$$
V=\frac{\lambda_{0} \Delta \varphi}{4 \pi \Delta t \cos \theta},
$$

where $V$ is the velocity resolution, $\Delta \varphi$ is the minimum detectable phase shift, $\Delta t$ is the A-line interval, $\theta$ is the Doppler angle (taken to be $180 \mathrm{deg}$ for velocity resolution), and $\lambda_{0}$ is the central wavelength of the OCT system. The SNR was obtained using methods outlined in Ref. 26. For reference, typical blood flow rates in chorioallantoic membrane (CAM) vessels ranging from 100 to $300 \mu \mathrm{m}$ in diameter have been measured to be between 1 and $8 \mathrm{~mm} / \mathrm{s}^{27,28}$ whereas blood flow rates in human vessels ranging from 0.8 to $1.8 \mathrm{~mm}$ in diameter were measured to be between 4.9 and $19.0 \mathrm{~cm} / \mathrm{s}$ in arteries and 1.5 and $7.1 \mathrm{~cm} / \mathrm{s}$ in veins. ${ }^{29}$

The entire optical payload, consisting of both high-power laser optics and OCT optics, is mounted onto a linear translation stage and a custom-built pitch and yaw stage, which was necessary due to the high angular sensitivity of the dichroic mirror. Exposure time was regulated using a THORLABS SC1 shutter controller and an SH1 single blade shutter, which was placed between the $1942 \mathrm{~nm}$ collimator and the dichroic mirror. Bulk alignment between the imaging and high-powered beams was accomplished using visible lasers. Alignment was verified at the start of each experiment by ablating moistened cardboard and ensuring that the resultant crater was within the imaging field of the 1300-nm beam using real-time OCT structural image feedback.

\section{Methodology}

All experiments were carried out according to recommendations provided by the research ethics board of the institution. A total of 80 avian egg embryos were cultivated and appropriately prepared for the experiment, which yielded 104 viable vessels. Each vessel was subjected to thulium laser irradiation, where exposure time, spot size, and average power were varied in order to generate a variety of outcomes. For each vessel, an OCT structural image, OCT Doppler image, and photograph were acquired for both pre- and postlaser exposure forming a single data point. Pre- and postimages were examined to evaluate the outcome.

\subsection{In-Ovo Specimen Cultivation}

Avian embryos were used in this study as they offer convenient access to functioning arteries and are a rapid, cost-efficient alternative to other in vivo models. Eggs were incubated in a custombuilt incubator. Access to embryonic arteries was achieved using a method outlined in Ref. 14 where the eggshell is breached in the vicinity of the air-pocket as to avoid damaging vital biological structures. Vessels were exposed by delicately peeling away the inner shell membrane from the CAM (Fig. 2).

In several preliminary experiments, avian embryos were allowed to develop between 5 and 18 days in order to determine the ideal age for Doppler imaging of CAM vessels. It was found that the optimal age for producing the highest number of viable vessels was between 10 and 12 days, where vessels were deemed viable if they were visible to the naked eye, embedded in the CAM, and small enough to be mostly within the imaging field of the OCT system. Embryos younger than 7 days were underdeveloped; vessels in the CAM membrane were typically not visible to the eye and air-pockets were not large enough to provide a sufficient working area. While 15-day old embryos produced many viable vessels, at this age embryos were well developed and tended to produce bulk motion artifacts in the Doppler signals. 


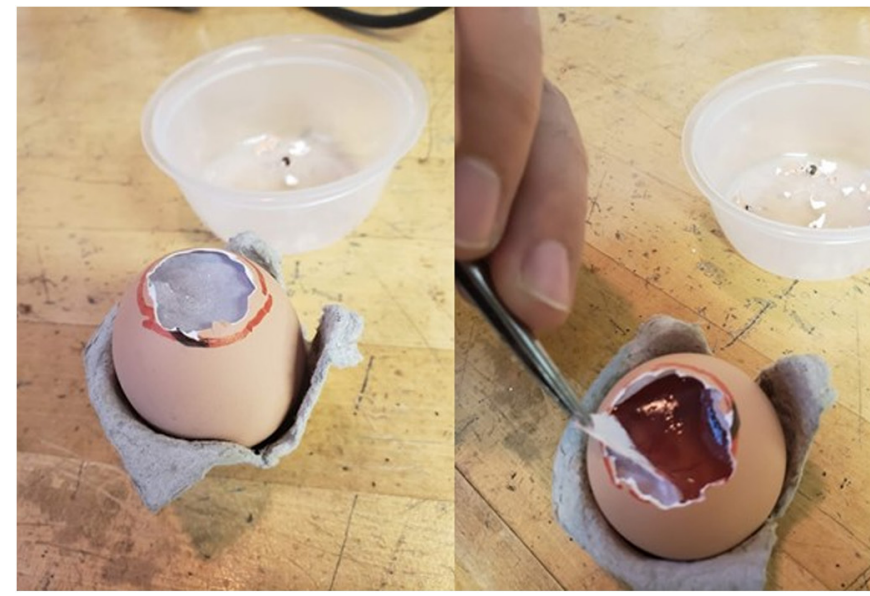

(a) (b)

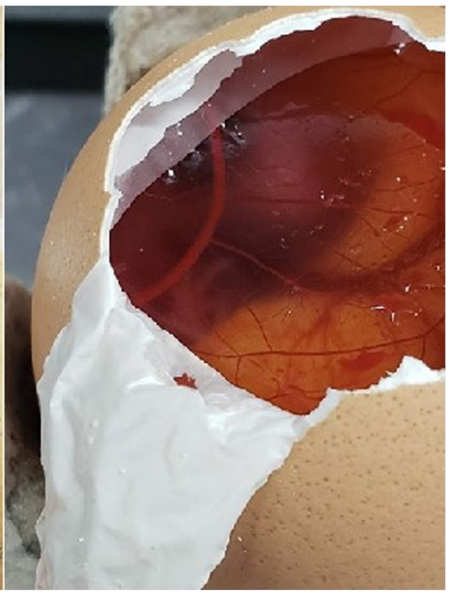

(c)

Fig. 2 Depiction of specimen preparation. (a) Egg shell removed in the viscinity of the airpocket exposing inner shell membrane. (b) Inner shell membrane separation from CAM. (c) Inner shell membrane shown separated from CAM.

\subsection{Experimental Procedure}

For each data point, a vessel was first photographed and scanned via OCT where $\sim 100$ B-scans were acquired at $6 \mathrm{~B}$-scans/s. B-scans were appropriately processed to generate both structural and Doppler images. Given that the heart rate of the avian embryo is between 2 and $4 \mathrm{~Hz}^{30}$ averaging was used in the processing of both OCT modalities, which ensured that flow variation due to systole and diastole would be filtered. The chosen vessel was targeted using a real-time OCT structural image and was subsequently irradiated with the $2-\mu \mathrm{m}$ laser. A postirradiation OCT scan was then conducted in an identical manner to the pre-irradiation scan and a photograph was acquired. Great care was taken to ensure that both pre- and post-OCT scans were acquired in the same cross-section. OCT evaluation of outcomes was performed by contrasting pre- and postirradiation OCT images (both Doppler and structural) and thus is abbreviated as DS-Eval. Several markers were of interest primarily the presence or absence of flow in the region of interest (ROI) as demarcated by the accompanying OCT structural image in the post-DOCT image. The ROI used for DS-Eval was the vessel lumen and the immediate area about the vessel including the layer of the CAM superficial to the vessel. In addition to this, markers such as vessel diameter and general morphology were taken into consideration. Visual evaluation (V-Eval) was performed by a trained neurovascular surgeon (C.R.P.) by examining only pre- and postirradiation photographs. A vessel was deemed visually sealed when there was no evidence of arterial luminal filling beyond the coagulated region. This also included arterial vessel extravasation. V-Eval was done independently (i.e., the investigator was blinded from the corresponding OCT images).

\subsection{Statistical Analysis}

The chi-squared statistical test of independence was used to determine if relationships between categorical data exist. Our null hypothesis is that no relationship exists between the variables of interest and that samples are independent. Our specific aim was to determine if coagulation status of the vessel (seal or no seal) after irradiation was related to the method of evaluation (DS or V). The null hypothesis that seal status is independent of the method of evaluation was tested at the $5 \%$ level.

\section{Results}

Outcomes were classified by DS-Eval into four groups: seal, rupture, partial seal, and unaffected. Each outcome was classified according to the presence of primary and secondary biomarkers. The presence of the primary marker was absolutely necessary to classify an outcome while the 
Marques et al.: Doppler optical coherence tomography for energy seal evaluation...

Table 1 Summary of markers used for DS-Eval.

\begin{tabular}{|c|c|c|}
\hline Classification & Structural OCT & Doppler OCT \\
\hline Seal & $\begin{array}{l}\text { Evidence of embolism in the post-ROI } \\
\text { Significant change in vessel morphology from } \\
\text { pre- to post-ROI }\end{array}$ & No signal present in the post-ROI \\
\hline Partial seal & $\begin{array}{l}\text { Vessel constriction } \\
\text { Evidence of partial embolism in postimage }\end{array}$ & $\begin{array}{l}\text { Signal area reduced by } 15 \% \text { or } \\
\text { more between pre- and post-ROIs }{ }^{\mathrm{a}}\end{array}$ \\
\hline Unaffected & $\begin{array}{l}\text { Vessel morphology mostly preserved between } \\
\text { pre- and post-ROls }\end{array}$ & $\begin{array}{l}\text { Less than } 15 \% \text { change in signal area } \\
\text { between pre- and post-ROls }\end{array}$ \\
\hline Rupture & $\begin{array}{l}\text { Vessel disappears in postimage (annihilated) }{ }^{\mathrm{a}} \text { OR } \\
\text { Vessel is not intact, evidence of wall disruption }{ }^{\mathrm{a}}\end{array}$ & $\begin{array}{l}\text { Doppler signal is present at } \\
\text { a vessel cross-section adjacent to } \\
\text { the irradiation site }\end{array}$ \\
\hline & Significant morphological change & Diffuse Doppler pattern present \\
\hline
\end{tabular}

aDenotes the primary marker.

presence of secondary markers served to refute or support the conclusions drawn from the primary marker. Table 1 shows a summary of markers used to classify vessel seals by DS-Eval.

The mean vessel diameter was $0.50 \pm 0.16 \mathrm{~mm}$. Of the 104 vessels irradiated in this study 30 were seals, 31 ruptured, 20 were partial seals, and 23 were unaffected by DS-Eval. In order to compare DS-Eval with V-Eval, the four groups generated by DS-Eval were generalized into seal and nonseal groups where the nonseal group included partial seals, ruptures, and unaffected outcomes. In this way, DS-Eval reported that $29 \%$ of vessels had sealed (30 seals and 74 nonseals) compared with V-Eval, which reported that $47 \%$ of vessels had sealed (49 seals and 55 nonseals). A true positive (TP) occurred when both DS and V-Eval resulted in a seal, a true negative (TN) occurred when both DS and V-Eval resulted in a nonseal, a false positive (FP) was declared when V-Eval resulted in a seal and DS-Eval resulted in a nonseal, and a false negative (FN) occurred when V-Eval resulted in a nonseal and DS-Eval resulted in a seal. Following this, V-Eval generated 27 TPs, 52 TNs, 22 FPs, and 3 FNs resulting in a sensitivity of $90 \%$, specificity of $70 \%$, and an overall accuracy of $76 \%$. It was found that whether the outcome was classified as sealed or notsealed was dependent on the classification methodology, and this was statistically significant $(p=0.01$ using a two-tailed chi-squared test with Yates correction). Table 2 gives the contingency table from which the chi-squared statistic was calculated. Further, it was found that V-Eval specificity was dependent on the type of nonseal classification (partial seal, rupture, or unaffected) and this finding was also significant ( $p<0.0001$, chi-squared test). Table 3 shows the contingency table from which the chi-squared value was calculated.

\subsection{Detailed Analysis of Biomarkers Used for DS-Eval}

Referring to Table 1, the primary marker used for classifying an outcome as a seal was the lack of a Doppler signal in the post-ROI. This was the case for nearly all outcomes that were classified as seals. There were some cases where although the Doppler signal had disappeared in the

Table 2 The association between seal evaluation method and outcomes.

\begin{tabular}{lccc}
\hline \hline & DS-Eval & V-Eval & Total \\
\hline Seals & 30 & 49 & 79 \\
Nonseals & 74 & 55 & 129 \\
Total & 104 & 104 & 208 \\
\hline \hline
\end{tabular}


Marques et al.: Doppler optical coherence tomography for energy seal evaluation...

Table 3 The association between V-Evals and DS-Eval nonseal groups.

\begin{tabular}{lccc}
\hline \hline & V seals & V nonseals & Total \\
\hline Rupture & 4 & 27 & 31 \\
Partial seals & 14 & 6 & 20 \\
Unaffected & 4 & 19 & 23 \\
Total & 22 & 52 & 74 \\
\hline \hline
\end{tabular}

post-ROI, the outcome was not classified as sealed given that secondary markers strongly indicated otherwise. An unexposed vessel was well characterized by a characteristic lemniscate visible in pre-irradiation ROI. This lemniscate has been detected in other studies using OCT on CAM vasculature. ${ }^{31}$ Thus, if such was the case where the Doppler signal disappeared, and the lemniscate morphology was retained, then the vessel would not be considered sealed. Completely sealed vessels both lacked a Doppler signal in the post-ROI and exhibited a significant change in morphology. A significant change in morphology was considered to have occurred if the characteristic lemniscate disappeared from pre- to poststructural ROI indicating that sufficient energy was delivered to morph the vessel lumen. The strongest structural marker for a seal was evidence of embolism. Embolism was identified by the recognition of a uniform structural signal apparent in the post-ROI, i.e., the characteristic lemniscate morphology was

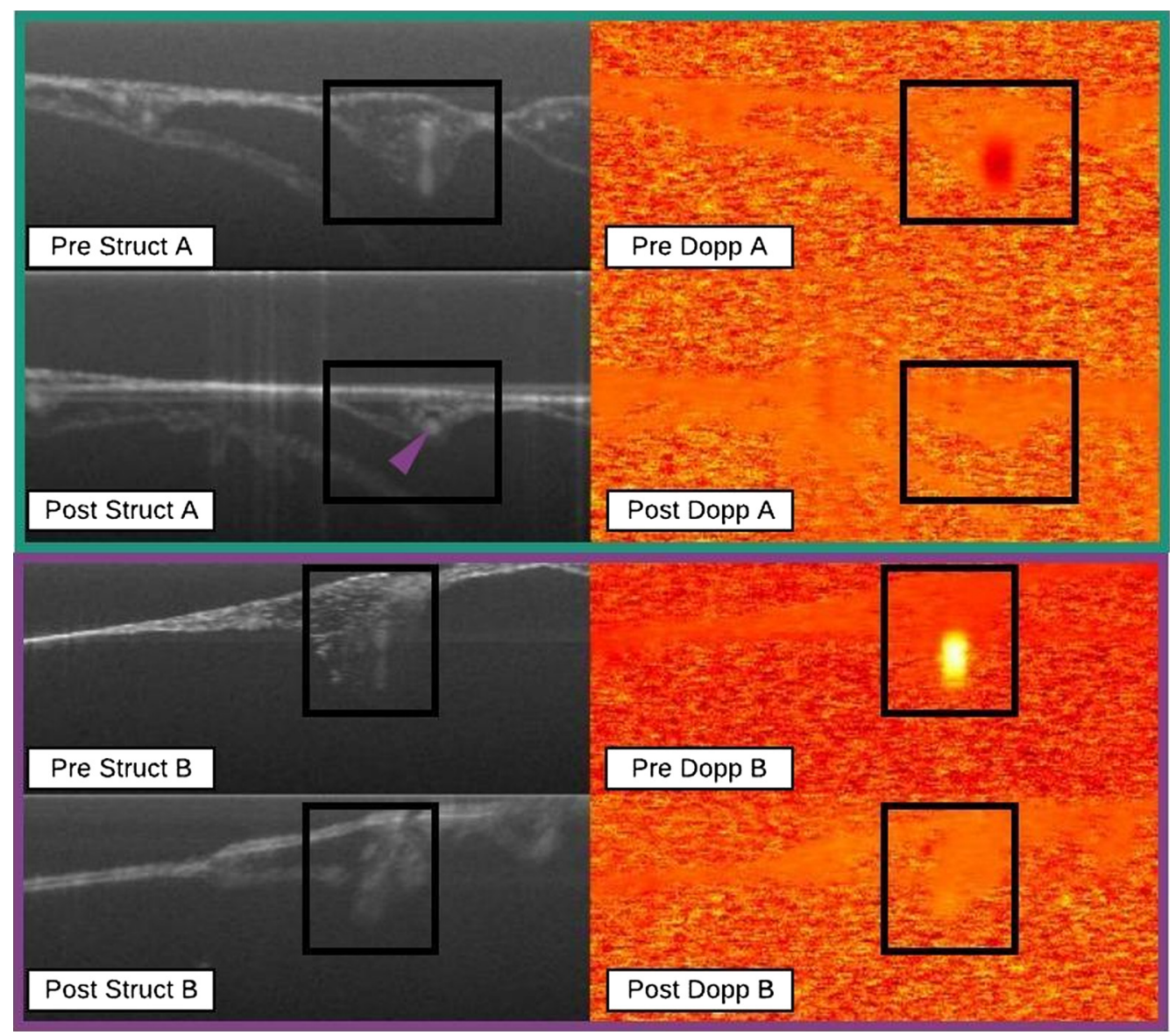

Fig. 3 Seal data points A and B. Evidence of embolism (green rectangle) and significant morphological change (purple rectangle). Black boxes represent ROls and the purple triangle indicates uniform structural signal within vessel lumen suggesting embolism. In all data points note the disappearance of the lemniscate morphology from pre- to poststructural images as well as the disappearance of the Doppler signal in the post-ROI. 
replaced by a homogeneous mass in the vessel lumen. The appearance of this structural marker and the accompanying lack of Doppler signal within the post-ROI indicates that a static structure has developed within the lumen thereby strongly suggesting embolism. Figure 3 gives examples of seal data points that exhibit both evidence of embolism and significant morphological change.

Partial seals were well defined by a decrease in Doppler signal area between pre- and postirradiation ROIs. To elucidate, a decrease in Doppler signal area meant that the number of pixels demonstrating Doppler signal in the ROI would decrease from pre- to post-Doppler images. In order to differentiate from the unaffected classification, at least a $15 \%$ decrease in Doppler signal area must be observed for an outcome to be classified as a partial seal. A change in the Doppler signal area directly indicates that some energy reached the lumen and affected blood flow. In some partial seals, the decrease in Doppler signal area was a result of vessel constriction induced by irradiation. Other cases involved partial embolism, where typically blood had coagulated in the most superficial portion of the vessel but not in the deeper portion. Figure 4 shows both vessel constriction and partial embolism.

The unaffected classification is a misnomer as many vessel irradiation outcomes in this category certainly exhibited some response to laser irradiation. A more appropriate title for this classification would have been "no significant hemostatic effect" but, for brevities sake, unaffected was used. A vessel was considered unaffected if vessel morphology was mostly preserved from pre- to postirradiation. This was apparent by comparing pre- and poststructural images. "Mostly preserved" indicates that the vessel diameter remained within $10 \%$ of its unexposed state and that the lemniscate morphology was present in the poststructural ROI. In addition,

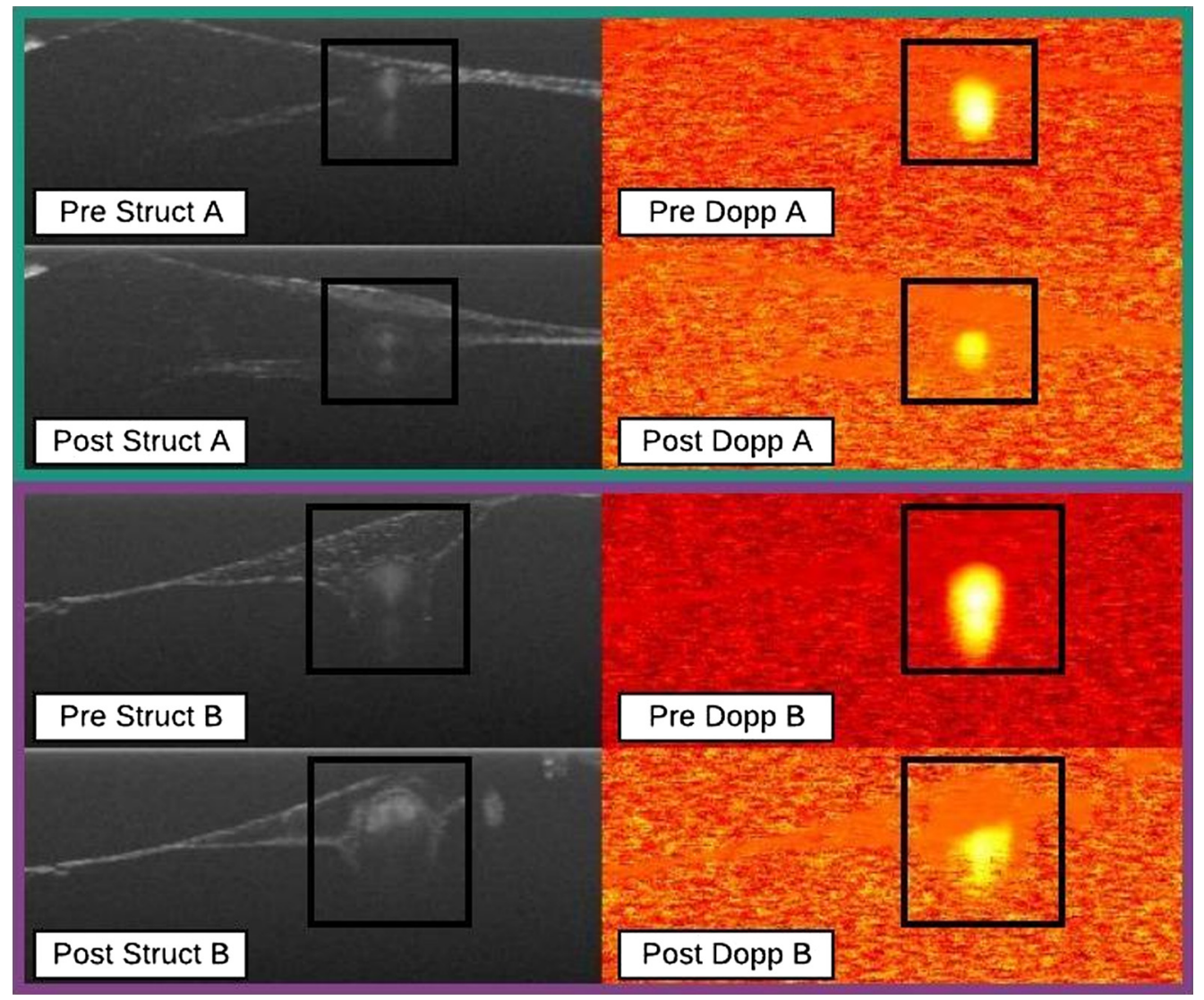

Fig. 4 Partial seal data points. Vessel constriction (A, green rectangle) and partial embolism ( $B$, purple rectangle). Black boxes indicate the ROls. In data point $A$, the Doppler signal area has clearly been reduced by more than $15 \%$ while vessel constriction is apparent from the change in vessel diameter from pre- to poststructural images. In data point B, the Doppler signal area has decreased by more than $15 \%$ while from the poststructural image, the homogeneous signal coming from the superficial portion of the vessel lumen accompanied by a lack of a Doppler signal indicates that partial embolism has been achieved. 


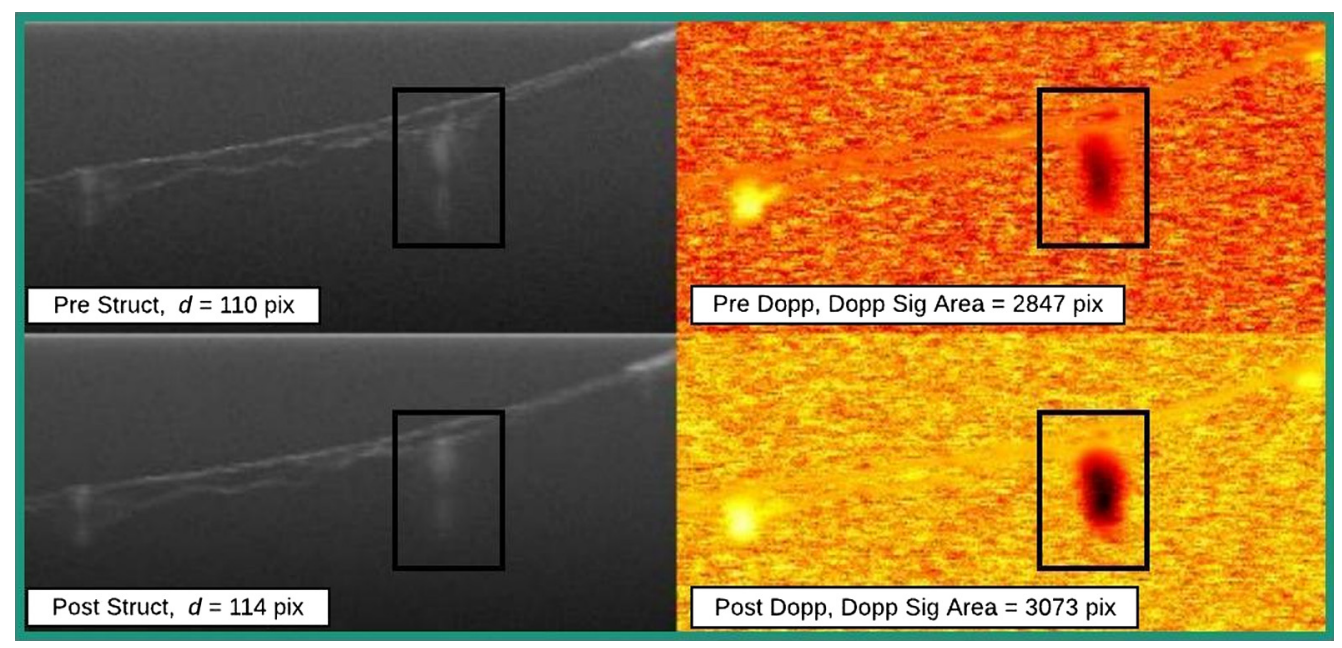

Fig. 5 An unaffected data point. Black boxes indicate the ROls. This data point exhibits $<10 \%$ change in vessel diameter and $<15 \%$ change in Doppler signal area and thus is classified as unaffected. Vessel diameters, $d$, are labeled in each structural image and are reported in pixels. Doppler signal area (Dopp Sig Area) is labeled in each Doppler image and is also given in pixels.

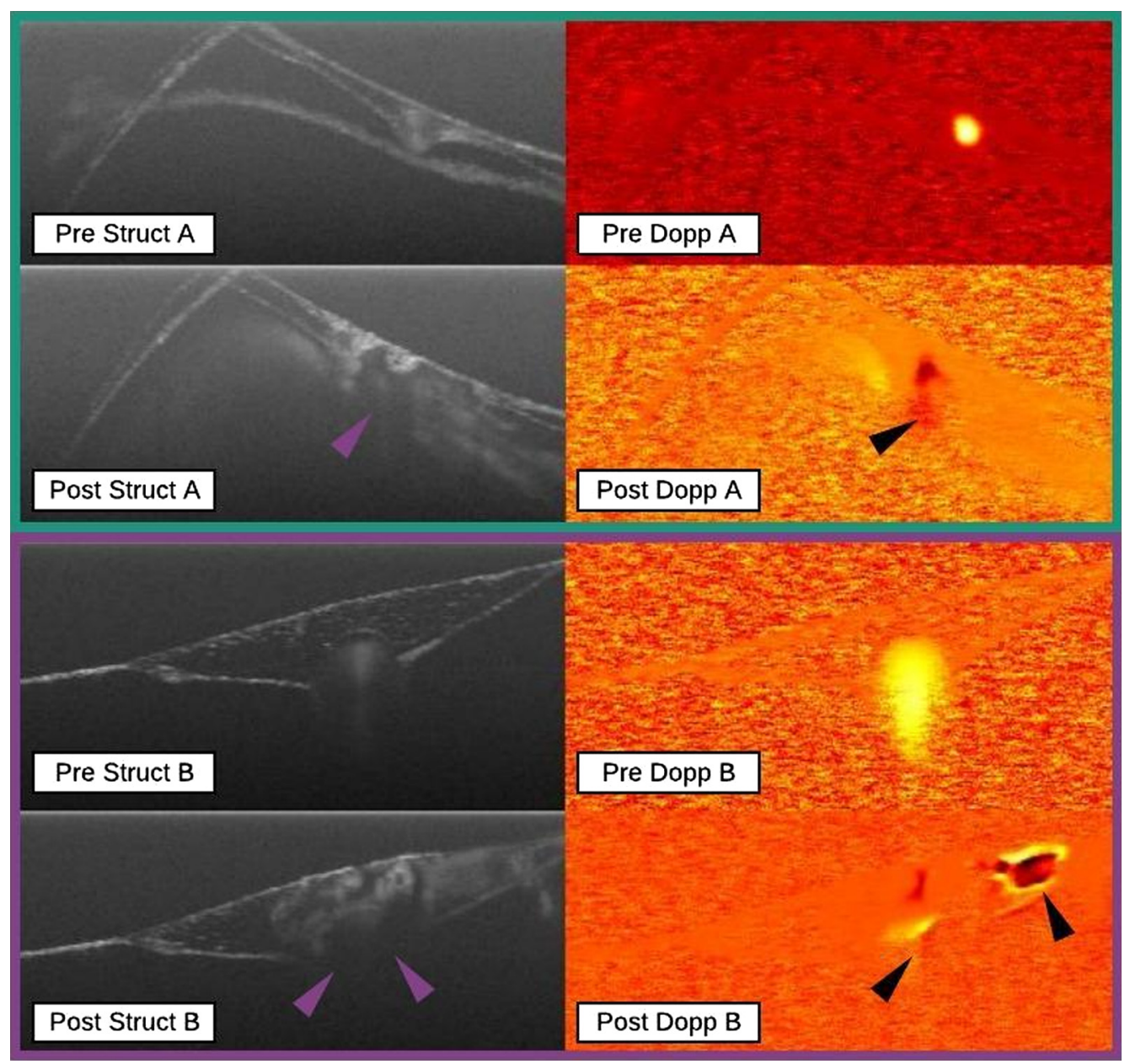

Fig. 6 Rupture data points. Both data points A (green rectangle) and B (purple rectangle) demonstrate wall breach indicated by purple arrows while the presence of the diffuse Doppler signal (black arrows) supports the rupture classification. ROls are left unlabeled for clarity. 
for an outcome to be classified as unaffected, the Doppler signal area would have to remain within $15 \%$ of its original size. Both the $10 \%$ limitation on diameter change and the $15 \%$ limitation on Doppler signal area change were arbitrarily chosen to distinguish between unaffected and partial seal outcomes. Here, the primary purpose of DS-Eval was to objectively classify outcomes as sealed or not sealed and thus, since both partial seal and unaffected are both nonseal outcomes, it is not crucial to have objective differentiation between the two categories. The thresholds delineating the two classifications may change to suit the specific needs of a given application of DS-Eval. Figure 5 gives an example of an unaffected data point.

Vessel rupture was declared whenever there was evidence of bleeding. Several markers were good indicators of bleeding especially vessel eradication, which was evident from structural images when the vessel was no longer within the field of view. Alternatively, bleeding was also evident when the structural images exhibited vessel wall disruption differentiated from complete annihilation as part of the vessel wall remained intact postirradiation. Secondary markers included the adjacent Doppler signal and the diffuse Doppler signal. The adjacent Doppler signal was acquired at an intact cross section of vessel directly beside the irradiation site. If Doppler signal was present here it was assumed that blood flow had not been ceased within the vessel and thus a rupture was probable. The diffuse Doppler signal was recognized when Doppler signal existed outside of the ROI indicating vascular extravasation. It is termed "diffuse" as the Doppler signal would often be spread over the image and not localized to the ROI. Figure 6 shows two rupture data points with labeled biomarkers.

\section{Discussion}

The primary objective of this study was to introduce Doppler and structural OCT for the evaluation of energy seals induced in small vessels by laser irradiation. The methodology developed termed DS-Eval uses specific biological markers listed in Table 1 to determine whether a vessel had been sealed. In general, the presence of these markers is not based on subjective opinion but rather on numerical information or clear imagery. Incidentally, DS-Eval is objective making it suitable for scientific processes and generating reliable results in the evaluation of laser sealing systems. Acquisition time of each data point was $\sim 15 \mathrm{~min}$ because the specimen had to be maneuvered into the imaging field of the OCT beam and a total of 200 B-scans (including pre- and postscans) were acquired at 6 B-scans/s. In some cases, movement of the embryo caused motion artifacts to appear in the Doppler images while structural images were at times ruined by brightness artifacts. In both cases, a rescan of the vessel was required and acquisition times were as long as $30 \mathrm{~min}$. The postprocessing time of each data point was $\sim 2.5 \mathrm{~min}$. Improvements to DS-Eval workflow could be realized by parallelizing the Doppler and structural OCT processing algorithms and upgrading supporting technology, primarily the devices used to maneuver the optical payload into position as this was the most time-consuming process.

Most clinical energy sealing devices use mechanical compression. ${ }^{32}$ Since contactless energy sealing was implemented in this study, it is important to address the potential influences of compression on the overall DS-Eval methodology. First, compressing the vessels before imaging would essentially eliminate the $3-\mathrm{mm}$ vessel diameter limitation posed by the limited optical penetration depth of the imaging beam. The use of DS-Eval could therefore be extended to larger vessels. Second, compression of the vessel would negate the utility of the lemniscate biomarker and many of the other structural biomarkers listed in Table 1. Irradiation outcomes would therefore be more-so dependent on Doppler biomarkers rather than structural biomarkers. The primary biomarker in compression based energy sealing used to delineate seals and nonseals would likely be the presence or absence of flow in the post-Doppler image ROI. Finally, since mechanical compression of the vessel would likely result in a temporary cessation of blood flow, postirradiation scans would have to be conducted once the compression has been released to ensure that flow has ceased due to vessel denaturation. Clinically available systems are not only capable of sealing individual vessels but are also able to seal vascular tissue bundles. In these cases, DS-Eval could still be utilized provided that the lateral width of the imaging window (defined by the galvo sweep range and the distance from focal lens to sample) could be made sufficiently wide to capture all vessels within the bundle. In compression-based approaches, 
it is assumed that the width of the bundle postirradiation would be significantly less than its width in the natural state. This meaning that the width of the imaging window may not have to be significantly increased in order to apply DS-Eval to vascular tissue bundles.

In this study, it was presumed that DS-Eval had an accuracy of $100 \%$. While this is certainly not absolute, the authors believe that the accuracy of DOCT is very near to the gold standard CT angiography given DOCT's high velocity resolution. Of course, there are several possible sources of error that could have potentially decreased the accuracy of DS-Eval such as user error (inability to detect failed scans resulting from motion and brightness artifacts) and inherent DOCT limitations. For example, the flow sensitivity of DOCT is related to the A-line rate and the Doppler angle, thus if there was a case where the vessel was scanned in such a way that the imaging beam was orthogonal to the flow direction DS-Eval would fail. This could be avoided by proper optical payload positioning relative to the vessel. In addition, it is also possible to encounter residual flows that are slower than the velocity resolution of the DOCT system, which would also result in a failed classification.

OCT used in this manner has also revealed a variety of biologically related insights into the energy sealing of vessels. Not only was it possible to determine whether the vessel had been sealed, but it was also possible to infer why. For example, by referring to Fig. 4B, it is evident that this vessel did not seal. Further, by observing both the structural and Doppler images, it can be concluded that the vessel did not seal because insufficient thermal energy reached the entirety of the vessel lumen. This conclusion is drawn since the superficial portion of the vessel seems to have been affected while the deeper portion was not. Based on this observation, perhaps vessel sealing using the $\mathrm{Tm}^{3+}$ laser could be improved by adjusting the focus of the beam into the center of the vessel lumen. In general, these in situ microscopic observations of vascular biomechanical response to laser irradiation are indispensable as they lend greater insight as to the mechanisms involved in laser sealing allowing for improvements to be made to laser sealing systems.

It was found that the outcomes seal or no seal and the evaluation method with which they were determined are dependent and this finding was statistically significant. Referring to Table 3 , it can be seen that $64 \%$ of FPs reported by V-Eval were partial seals. Subsequently, the specificity of V-Eval was found to be strongly dependent on the type of nonseal classification and this finding was also statistically significant. Therefore, it is reasonable to conclude that in laser sealing studies where V-Eval was used to evaluate energy seals, reported seal rates are likely inflated. Further, this inflation is likely a result of an inability to differentiate partial seals from seals by eye. Meticulous hemostasis is paramount for all surgical procedures, and thus all coagulated vessels should be completely sealed. Partial seals would pose a significant risk, especially if partial seals resulted in slow ooze from the vessel that is not detected visually at the time of surgery. Delayed postsurgical hematomas are not uncommon, and it is reasonable to assume these may be due to incompletely sealed vessels. ${ }^{33}$ In intracranial surgeries, the percentage of postsurgical hematomas requiring surgical intervention ranges from $0.8 \%$ to $6.9 \%$. $^{34,35}$ Thus, current laser technologies used for the purpose of vessel sealing, which were evaluated visually should be reevaluated to ensure that complete vessel seals were achieved.

DS-Eval of vessel seals is not limited solely to laser-based systems, it can be extended for use across all energy sealing systems increasing its utility. Its limitation imposed by a limited penetration depth is compensated for by its high velocity resolution and valuable structural feedback. Further, DS-Eval can be used in the seal classification of large vessels if used intravascularly, which is already a commonly used technique for the evaluation of blood clots. ${ }^{36}$ Here, DS-Eval was presented as a benchtop testing method for laser-based energy sealing systems. Clinical translation of this methodology will not be possible without significant improvement to supporting technology, validation of DS-Eval through comparison with an existing methodology such as CT angiography, and overall modification of biomarkers to suit human anatomy. The system should be modified such that the sample arm is fitted into a handheld contact-based probe similar to existing clinical ultrasound systems. This would potentially reduce acquisition times by up to $15 \mathrm{~min}$ since payload maneuvering was the most timeconsuming process. In the literature, B-scan postprocessing rates have been reported to be as fast as 40 FPS when GPU-FPGA integrated systems were used. ${ }^{37}$ If these frame rates were implemented here, then the postprocessing times could be decreased from $2.5 \mathrm{~min}$ to $5 \mathrm{~s}$. Based on these improvements to supporting technology, it is estimated that the overall acquisition and 
processing time for a single data point could be performed in under a minute. The primary biomarker used for energy seal evaluation here was the characteristic lemniscate that defined the natural state of the CAM vessel. This lemniscate is not common to human anatomy and thus a new biomarker defining the natural state of human vasculature must be defined. Further, human vessel structure is not constant across all vessel types. For example, arteries proximate to the heart are elastic and feature thinner walls with larger lumina, whereas arteries distal from the heart are muscular with smaller lumina and thicker walls. ${ }^{38}$ Therefore, for clinical translation of DS-Eval, it may be necessary to redefine the natural state biomarker for several vessel types.

\section{Conclusion}

An objective methodology employing both Doppler and structural OCT feedback was introduced for the purposes of energy seal evaluation. The importance of introducing such a methodology is for both evaluating the hemostatic potential of energy sealing systems and for the noninvasive confirmation of hemostasis. Although acquisition and processing times reported here are quite lengthy, this was primarily a feasibility study; clinical translation of this technology can be realized by improving processing speeds and overall ease of use. In general, DS-Eval has the potential to be an indispensable method for the evaluation of energy seals not solely due to its high velocity resolution but also due to valuable microscopic morphological insight regarding the biological mechanisms involved in energy sealing. Future studies will involve dynamic structural and Doppler imaging during laser irradiation to visualize the sealing process.

\section{Disclosures}

The authors declare that there are no conflicts of interest related to this article.

\section{Acknowledgments}

The authors thank the Canada Research Chair Program of the Natural Sciences and the Engineering Research Council of Canada.

\section{References}

1. S. R. Chandra, "Intraocular tumors: a text and atlas," Arch. Ophthalmol. 112(2), 169 (1994).

2. J. A. Shields, C. L. Shields, and P. De Potter, "Photocoagulation of retinoblastoma," Int. Ophthalmol. Clin. 33(3), 95-99 (1993).

3. J. A. Burns et al., " $532 \mathrm{~nm}$ pulsed potassium-titanyl-phosphate laser treatment of laryngeal papillomatosis under general anesthesia," Laryngoscope 117(8), 1500-1504 (2007).

4. S. M. Zeitels et al., "Office-based 532-nm pulsed KTP laser treatment of glottal papillomatosis and dysplasia," Ann. Otol. Rhinol. Laryngol. 115(9), 679-685 (2006).

5. S. M. Zeitels et al., "Pulsed angiolytic laser treatment of ectasias and varices in singers," Ann. Otol. Rhinol. Laryngol. 115(8), 571-580 (2006).

6. J. A. Werner et al., "Current concepts in the classification, diagnosis and treatment of hemangiomas and vascular malformations of the head and neck," Eur. Arch. Oto-RhinoLaryngol. 258(3), 141-149 (2001).

7. J. A. Werner et al., "Ultrasound-guided interstitial Nd: YAG laser treatment of voluminous hemangiomas and vascular malformations in 92 patients," Laryngoscope 108(4 $\mathrm{Pt} 1)$, 463-470 (1998).

8. R. R. Anderson and J. A. Parrish, "Selective photothermolysis: precise microsurgery by selective absorption of pulsed radiation," Adv. Sci. 220(4596), 524-527 (1983).

9. C. M. Cilip et al., "Infrared laser thermal fusion of blood vessels: preliminary ex vivo tissue studies," J. Biomed. Opt. 18(5), 058001 (2013).

10. N. C. Giglio et al., "Rapid sealing and cutting of porcine blood vessels, ex vivo, using a high-power, 1470-nm diode laser," J. Biomed. Opt. 19(3), 038002 (2014).

11. L. A. Hardy et al., "Rapid sealing of porcine renal blood vessels, ex vivo, using a high power, 1470-nm laser, and laparoscopic prototype," J. Biomed. Opt. 22(5), 058002 (2017). 
12. S. Kimel et al., "Vascular response to laser photothermolysis as a function of pulse duration, vessel type, and diameter: implications for port wine stain laser therapy," Lasers Surg. Med. 30(2), 160-169 (2002).

13. C. J. Rees et al., "Effects of gold laser on the avian chorioallantoic membrane," Ann. Otol. Rhinol. Laryngol. 119(1), 50-53 (2010).

14. M. S. Broadhurst et al., "Effects of $532 \mathrm{~nm}$ pulsed-KTP laser parameters on vessel ablation in the avian chorioallantoic membrane: implications for vocal fold mucosa," Laryngoscope 117(2), 220-225 (2007).

15. C. M. Cilip et al., "Infrared laser sealing of porcine vascular tissues using a 1,470 nm diode laser: preliminary in vivo studies," Lasers Surg. Med. 49(4), 366-371 (2017).

16. J. A. Burns et al., "Predicting clinical efficacy of photoangiolytic and cutting/ablating lasers using the chick chorioallantoic membrane model: implications for endoscopic voice surgery," Laryngoscope 118(6), 1109-1124 (2008).

17. A. Major et al., "Microvascular photodynamic effects determined in vivo using optical Doppler tomography," IEEE J. Sel. Top. Quantum Electron. 5(4), 1168-1175 (1999).

18. W. Drexler and J. G. Fujimoto, Optical Coherence Tomography: Technology and Applications, 2nd ed., Springer International Publishing, Switzerland (2015).

19. S. L. Bridgers, W. Haven, and L. Success, "Clinical correlates of Doppler/ultrasound errors in the detection of internal carotid artery occlusion," Stroke 20(5), 612-615 (1989).

20. Y. Huang et al., "Pulsed and CW adjustable $1942 \mathrm{~nm}$ single-mode all-fiber Tm-doped fiber laser system for surgical laser soft tissue ablation applications," Opt. Express 24(15), 16674 (2016).

21. A. R. Miller et al., "Comparison between spectral-domain and swept-source optical coherence tomography angiographic imaging of choroidal neovascularization," Investig. Ophthalmol. Vis. Sci. 58(3), 1499-1505 (2017).

22. K. K. Shung, "High frequency ultrasonic imaging," J. Med. Ultrasound 17(1), 25-30 (2009).

23. D. P. Popescu et al., "Optical coherence tomography: fundamental principles, instrumental designs and biomedical applications," Biophys. Rev. 3(3), 155-169 (2011).

24. A. Heimdal and H. Torp, "Ultrasound Doppler measurements of low velocity blood flow: limitations due to clutter signals from vibrating muscles," IEEE Trans. Ultrason. Ferroelectr. Freq. Control 44(4), 873-881 (1997).

25. X. Xu et al., "High-frequency ultrasound Doppler system for biomedical applications with a 30-MHz linear array," Ultrasound Med. Biol. 34(4), 638-646 (2008).

26. C. Chen et al., "Buffer-averaging super-continuum source based spectral domain optical coherence tomography for high speed imaging," Biomed. Opt. Express 9(12), 6529 (2018).

27. Z. Chen et al., "Noninvasive imaging of in vivo blood flow velocity using optical Doppler tomography," Opt. Lett. 22(14), 1119 (1997).

28. A. Davis, J. Izatt, and F. Rothenberg, "Quantitative measurement of blood flow dynamics in embryonic vasculature using spectral Doppler velocimetry," Anat. Rec. Adv. Integr. Anat. Evol. Biol. 292(3), 311-319 (2009).

29. M. Klarhö Fer et al., "High-resolution blood flow velocity measurements in the human finger," Magn. Reson. Med. 45, 716-719 (2001).

30. R. Akiyama et al., "Long-term measurement of heart rate in chicken eggs," Comp. Biochem. Physiol. A. Mol. Integr. Physiol. 124(4), 483-490 (1999).

31. X. Liu et al., "Spectroscopic-speckle variance OCT for microvasculature detection and analysis," Biomed. Opt. Express 2(11), 2995-3009 (2011).

32. S. D. Lyons and K. S. K. Law, "Laparoscopic vessel sealing technologies," J. Minimally Invasive Gynecol. 20(3), 301-307 (2013).

33. A. Nanda and D. P. Patra, 5-Surgical complications in neurosurgery, in Complications in Neurosurgery, A. Nanda, Ed., Elsevier Inc. (2019).

34. W. A. S. Taylor et al., "Timing of postoperative intracranial hematoma development and implications for the best use of neurosurgical intensive care," J. Neurosurg. 82(1), 48-50 (1995).

35. I. H. Kalfas and J. R. Little, "Postoperative hemorrhage: a survey of 4992 intracranial procedures," Neurosurgery 23(3), 343-347 (1988). 
36. M. J. Gounis et al., "Intravascular optical coherence tomography for neurointerventional surgery," Stroke 50(1), 218-223 (2019).

37. K. C. Jin, K. S. Lee, and G. H. Kim, "High-speed FPGA-GPU processing for 3D-OCT imaging," in 3rd IEEE Int. Conf. Comput. and Commun., (ICCC 2017), pp. 2085-2088 (2018).

38. C. Vanputte et al., Seeley's Anatomy and Physiology, McGraw-Hill College, New York (2016).

Andrew J. Marques is a biomedical engineer currently working towards a $\mathrm{PhD}$ at the Institute of Medical Science, University of Toronto. He received his BEng in biomedical engineering in 2016 and MASc in biomedical engineering at Ryerson University in 2019. His research interests include optical coherence tomography for vascular tissue imaging, the application of $2 \mu \mathrm{m}$ laser for neural tissue ablation and hemostasis, surgical robotics, and automated vertebral fusion techniques.

Robnier Reyes is a biomedical engineer currently working towards a $\mathrm{PhD}$ in biomedical engineering at Ryerson University. He received his BEng in biomedical engineering in 2016 and MASc in biomedical engineering at Ryerson University in 2019. His research interests include optical coherence tomography for robotic feedback control, surgical robotics, and automated vertebral fusion techniques.

Christopher R. Pasarikovski received his honors BSc degree in medical physics at the University of Western Ontario before gaining acceptance into medical school. He completed medical school in 2015 at Western, and is currently a fourth-year neurosurgical resident at the University of Toronto. Following his third year of residency, he entered the surgeon-scientist training program and is currently enrolled as a $\mathrm{PhD}$ candidate in the Institute of Medical Science. His research focus is the application of endovascular optical coherence tomography imaging in cerebrovascular disease.

Chaoliang Chen received his bachelor's and $\mathrm{PhD}$ degrees in optical engineering from Nanjing University of Science and Technology, and won the First Prize Scholarship of Nanjing University of Science and Technology for his research. He is currently a post doctoral fellow at the Tearney Lab, Massachusetts General Hospital, developing novel optical coherence tomography applications for clinical intervention.

Joel Ramjist is a researcher at Ryerson University, and has managed the Biophotonics and Bioengineering Laboratory since 2015. His research interests include optical coherence tomography and related angiographic methods for study of clinical pathologies, 3-D modeling and rapid prototyping for patient-specific phantom fabrication, intraoperative image-guided therapeutics including fluorescence and micro-ultrasound, and applications of augmented reality for surgical intervention.

Xijia Gu received his BSc degree from Nankai University, Tianjin, China, in physics, in 1982, his MSc degree from the University of Toronto, Toronto, Ontario, Canada, in 1984, and his PhD from the University of Waterloo, Waterloo, Canada, in 1987. He worked as a research fellow at Max Planck Institut für Strömungsforschung, Goettingen, German, and University of Toronto between 1987 and 1990. In 2003, he joined the Department of Electrical and Computer Engineering, Ryerson University, as an associate professor. He has published 91 referred journal articles, 39 conference papers, and holds four patents.

Victor Yang is a leader in translational medicine-specifically for his work in moving optical technologies to active use in the operating room to improve surgical practice. He received his BASc in engineering science, biomedical option, from the University of Toronto in 1997, and completed a master's in electrical and computer engineering in 1998. Dr. Yang entered the MD$\mathrm{PhD}$ program at the University of Toronto, completing his $\mathrm{PhD}$ in the Department of Medical Biophysics in 2004 for his work in Doppler optical coherence tomography, and completed his MD in 2006. 